\title{
Análisis complejo y transdisciplinar del enclaustramiento marítimo boliviano
}

\section{Karina Shirley Vera Quezada}

Karinaveraquezada@gmail.com

kveraq@post.emi.edu.bo

Licenciatura en Auditoria (UMSS), Magister en

Gestión Empresarial (UMSS), Magister en Educación

Superior Universitaria (EMI), Doctorante del Doctorado en Educación con Enfoque en Complejidad y la Investigación Transdisciplinar (EMI), Doctorante del doctorado en Ciencias Empresariales (UAB)

$76919994-70719568$

Cochabamba - Bolivia

\section{Dorian Ariel Arce Conde} arielarcecond@hotmail.com

darcec@post.emi.edu.bo

Oficial Militar Ejercito de Bolivia, en el grado de Teniente Coronel, realizo estudios en la Escuela Militar de Ingeniería en la carrera de Ingeniería geografía, Magister en Metodología de la Investigación Científica

y Educación Superior, Cursante de la maestría en Seguridad, defensa y desarrollo del Estado en la EAEN y del Doctorado en Complejidad y Educación Transdisciplinar DOE-6 EMI. 71531517-71531516

\section{RESUMEN}

Dentro de las políticas y estrategias para buscar una salida soberana al mar de Bolivia, desde la perspectiva dialógica está la Historiología que viene a ser el análisis estratégico de la historia que, difiere con la Historiografía que se ocupa de la cronología histórica, por esa razón en el presente artículo se desarrolla un análisis desde la complejidad para dar a conocer sobre la política y estrategia que debe emplear Bolivia en el tema marítimo tomando la perspectiva compleja y transdisciplinar analizando la multidimensionalidad de opciones y por ello se puede decir que, "Chile no es el único camino en que Bolivia podría confluir para lograr acceder a un puerto y costas 
marítimas". Por esta razón, para el presente análisis, según (Cruz Barreiro, 2009) Morín explicó que: será necesario considerar que el ser humano es a la vez físico, biológico, psíquico, cultural, social e histórico y esta unidad compleja de la naturaleza humana que está desintegrada y debe ser restaurada de tal manera que desde donde esté, tome conocimiento y conciencia de su identidad compleja y de su identidad común a todos los demás humanos.

A lo largo de las últimas décadas las condiciones de la política internacional expuesta por el estado de Bolivia, fueron más agresivas y tuvo un impacto a nivel internacional, tal es el caso de la demanda ante la Haya para obligar al estado Chileno a una negociación que permita una salida soberana al océano pacifico. (Morin \& Le Moigne, La inteligencia de la complejidad Epistemología y Pragmática, 2006) hacía referencia a lo siguiente: "Como decía Pascal, hay que concebir la relación circular: no se pueden conocer las partes si no se conoce el todo, ni se puede conocer el todo sin conocer las partes. La noción de organización se vuelve capital porque, por medio de la reorganización de las partes en un todo, aparecen las cualidades emergentes y desaparecen las inhibidas". Entonces será necesario religar esta política a partir de múltiples nociones de orden, desorden, organización y reconocer los problemas de irreducibilidad e indeducibilidad de relaciones complejas existentes entre las partes y el todo para luego unir la noción de unidad con la de pluralidad o diversidad y de este modo llegar a confluir en probables acuerdos.

Por no contar con una política de estado que integre los diferentes niveles de conducción y factores de poder a través de una defensa multidisciplinaria con un enfoque integrado de las dimensiones de poder, el planteamiento que realizó Bolivia no fue el adecuado por lo que se puede decir que, la decisión de la corte no fue la esperada, lo que tuvo un impacto dentro de la moral social por las expectativas que se habían creado, por lo citado el presente artículo hace un análisis complejo desde una visión multidimensional dando paso a un modo de conocimiento capaz de aprehender los objetos en sus contextos y a la vez en un conjunto, reuniendo y organizando conocimientos dispersos y de esta manera mostrar la unión indisoluble entre la unidad y la diversidad de todo lo que es humano.

Palabras claves: enclaustramiento; políticas y estrategias; soberanía; acceso marítimo; complejidad; transdisciplinariedad; multidimensional 
Vera Quezada y Arce Conde...

\title{
Complex and transdisciplinary analysis of confinement Bolivian maritime
}

\begin{abstract}
Within the policies and strategies to seek a sovereign exit to the sea of Bolivia, from the dialogic perspective is the Historiology that becomes the strategic analysis of history that differs from the Historiography that deals with the historical chronology, for that reason In this article, an analysis from complexity is developed to make known about the policy and strategy that Bolivia should employ in the maritime issue, taking a complex and transdisciplinary perspective, analyzing the multidimensionality of options and therefore it can be said that, "Chile does not it is the only way in which Bolivia could converge to gain access to a port and maritime coasts". For this reason, for the present analysis, according to (Cruz Barreiro, 2009) Morín explained that: it will be necessary to consider that the human being is at the same time physical, biological, psychic, cultural, social and historical and this complex unit of human nature that it is disintegrated and must be restored in such a way that from where it is, it becomes aware and aware of its complex identity and its common identity to all other humans.

Throughout the last decades, the conditions of international policy exposed by the state of Bolivia were more aggressive and had an impact at the international level, such is the case of the lawsuit before The Hague to force the Chilean state to negotiate that allow a sovereign exit to the Pacific Ocean. (Morin \& Le Moigne, The intelligence of complexity Epistemology and Pragmatics, 2006) referred to the following: "As Pascal said, you have to conceive the circular relationship: you cannot know the parts if you do not know the whole, nor you can know the whole without knowing the parts. The notion of organization becomes capital because, through the reorganization of the parts into a whole, emergent qualities appear and inhibited ones disappear". Then it will be necessary to reconnect this politics from multiple notions of order, disorder, organization and to recognize the problems of irreducibility and indeducibility of complex relationships existing between the parts and the whole to later unite the notion of unity with that of plurality or diversity and of In this way, they come together in probable agreements.
\end{abstract}


Because it does not have a state policy that integrates the different levels of leadership and power factors through a multidisciplinary defense with an integrated approach to the dimensions of power, the approach made by Bolivia was not adequate from what can be said. that, the court's decision was not the expected one, which had an impact on social morality due to the expectations that had been created, therefore, this article makes a complex analysis from a multidimensional perspective, giving way to a way of knowledge capable of apprehending objects in their contexts and at the same time in a set, gathering and organizing dispersed knowledge and in this way showing the indissoluble union between unity and diversity and all that is human

Keywords: enclosure; policies and strategies; sovereignty; maritime access; complexity; transdisciplinarity; multidimensional

Artículo recibido: 15 octubre. 2021 Aceptado para publicación: 18 noviembre 2021 Correspondencia: Karinaveraquezada@gmail.com Conflictos de Interés: Ninguna que declarar 
Vera Quezada y Arce Conde...

\section{INTRODUCCION}

Dentro de la historia de Bolivia, una de las páginas más amargas que tocó vivir está la pérdida de su salida soberana al océano pacifico, producto de la ambición del vecino país de Chile que, a través de una guerra tripartita usurpó territorios soberanos que no pudieron ser resueltos desde la dimensión política, lo que ocasionó el enclaustramiento marítimo tan fundamental para el desarrollo económico de un País. Fueron varios los intentos de negociaciones que se realizaron, las mismas que, denotaron en falsas promesas y fallidos intentos de negociaciones mismos que, en el trascurso del tiempo solo trajeron el lamento y la discordia entre ambos países los cuales, hasta hoy mantienen esta problemática sin una aproximación de solución. Para (Morin, 1999), el ser humano es a la vez físico, biológico, psíquico, cultural, histórico y al ser una unidad compleja, es necesario comprender la multidimensionalidad de puntos de vista de los diferentes países ya que al comprender la complejidad de las perspectivas, priman intereses y la propia cultura al momento de asumir determinaciones que beneficien a los actores ya que cada individuo, a partir de su posición, debe tomar conocimiento y conciencia de su identidad compleja y común que la caracterizan del resto. Por tanto, en el presente análisis, es necesario considerar la condición humana emergente como objeto esencial para cualquier negociación.

Por consiguiente, ante los hechos acontecidos, se produjo la demanda por parte de Bolivia ante instancias de la Haya y el fallo fue que Chile no tiene la obligación de negociar con Bolivia una salida soberana al mar de acuerdo con la Corte Internacional de Justicia, por tanto, se debe buscar una forma de entablar un diálogo sobre este asunto entre ambas naciones para llegar a un buen acuerdo. Por parte de Bolivia, el actual presiente Lic. Luis Arce Catacora hace referencia que, la demanda no establece con claridad en su fallo que queda cerrada toda negociación y que Bolivia no desistiría de su aspiración legítima a su salida soberana, abriendo nuevamente la posibilidad de un debate que permita aprehender los objetos en sus contextos, sin dejar de lado sus complejidades y sus conjuntos sin dejar de lado los intereses de los diversos actores.

\section{ESTRATEGIAS METODOLOGICAS}

El presente artículo académico de análisis crítico ha sido desarrollado en Bolivia a partir de una revisión y síntesis de información recopilada a cerca de los avances del enclaustramiento marítimo de Bolivia a partir de un análisis cronológico 
multidimensional con un enfoque complejo y transdisciplinar para luego abordar en una nueva visión de posibles alternativas que proporcionan perspectivas de salidas a la mediterraneidad de Bolivia al mar.

\section{RESULTADOS}

\section{Análisis complejo y multidimensional de posibles salidas a nuestra mediterraneidad.}

Ante la situación actual que se vive en la actualidad, se puede indicar que, Bolivia no cuenta con una Política de estado claramente definida y solo se promueve una política de gobierno que desarrolla acciones gestionarias sin proyección futura, denotando intereses fragmentados que no permiten lograr el vínculo entre las partes intervinientes y las totalidades. Lo citado nos da a entender el por qué en las diferentes oportunidades históricas de un acceso soberano al océano no tuvieron un desarrollo favorable. Cabe mencionar que, dentro de las posibilidades que Bolivia tiene para salir del enclaustramiento fueron exploradas e incluso avanzadas varias hipótesis para que Bolivia recupere una salida al mar. Estas posibilidades son poco conocidas por que no se difundieron de manera general o en su defecto, no tuvieron la importancia en el ámbito político y social y tampoco fueron consideradas la multiplicidad de perspectivas que permitan reconocer la unidad y complejidad humana, permitiendo recoger y organizar conocimientos y necesidades dispersas que permitan mostrar la unión indisoluble entre lo que apetecen unos y también los otros representantes. En (apertura, 2011) la Cumbre de América Latina y del Caribe (CALC) de 2011, el presidente Evo Morales en su discurso de apertura, hizo un llamado a los "hermanos presidentes a gestar una gran integración", puntualizando: "Bolivia es un país pacifista que busca tener relaciones con todos los países del mundo que tiene la voluntad de participar en todos los procesos de integración" III Cumbre de Jefes de Estado y de gobierno de América Latina y el Caribe. 2011.

Como hace referencia (Longaric Rodriguez, 2014), la historia registra importantes esfuerzos diplomáticos bilaterales en 1950, 1961, 1975, 1986; como también gestiones multilaterales cuyos resultados constituyen derechos adquiridos e irrenunciables, como son las sendas resoluciones de las Asambleas generales de la OEA de 1979 y 1983. La Cancillería chilena en diversas etapas del diálogo bilateral, así como en el espacio multilateral, manifestó públicamente su intención de apoyar una salida al mar para Bolivia. Los pronunciamientos chilenos, respecto de esto, unas veces como oferta y 
otras como promesa, son expresiones que constituyen manifestaciones unilaterales con efectos vinculantes para las partes aludidas.

A continuación, se desglosan estas opciones:

\section{El puerto de Ilo en Perú}

En el año 1992, durante el gobierno de Paz Zamora fue anunciada la solución más ambiciosa y realista al enclaustramiento de nuestro país en el que los gobiernos de Bolivia y Perú firman un tratado por el cual Perú sede a Bolivia el puerto de Ilo, que representa una franja costera de cinco kilómetros al sur del territorio peruano, la misma fue cedida por 99 años, casi 27 años después, el sueño del puerto nunca llegó a despegar y la playa jamás generó turismo, generando un acontecimiento incierto e inesperado. Es así que, Bolivia Mar, lleva 26 años en el abandono por el escaso avance en las políticas de estado, como dice (Morin, 1999) ahondando más aún la incertidumbre de nuestros

tiempos y generando una incomprensión entre próximos y extraños, desde sus raíces, modalidades y efectos, centrándose en los racismos, xenofobias y desprecios.

\section{Puerto Busch-Paraguay}

Una alternativa todavía más antigua para Bolivia es una salida al Atlántico gracias a un tratado firmado con Paraguay en 1937 después de la guerra suscitada con este país en el cual se sede los territorios del chaco Boreal y en una cláusula se indica el derecho Boliviano al uso de las aguas del río para la salida al océano atlántico donde las embarcaciones bolivianas pueden alcanzar el mar partiendo desde sus propios muelles tras cruzar el río Paraguayo, desembocar en el Océano. Para (Morin, 1999) es necesario establecer una relación de control mutuo entre la sociedad y los individuos por medio de la democracia y concebir la humanidad como comunidad planetaria, por tanto, es importante impulsar la toma de conciencia de la tierra - patria, permitiendo que la misma sea traducida en la voluntad de realizar la ciudadanía terrenal. Para tal efecto, los bolivianos construyeron Puerto Busch aunque su puesta en marcha ha sido lenta, esta se encuentra sobre la frontera con Paraguay, en el departamento de Santa Cruz. Estos muelles bolivianos se encuentran en una zona estratégica porque Bolivia dispone a explotar minerales y ampliar sus servicios ferroviarios, por ello los expertos señalan que es necesario impulsar el crecimiento de Puerto Busch. 


\section{Argentina, Brasil y Uruguay}

A través de diferentes tratados, Argentina, Brasil y Uruguay le concedieron facilidades a Bolivia para la salida de sus productos por sus costas. Se trata de opciones de instalación de zonas libres de impuestos para los productos por esos países incluso facilidades en diferentes puertos, incluso el gobierno boliviano ha reconocido que no se han aprovechado de manera óptima estas concesiones obtenidas a lo largo de las últimas décadas. Como dice (Morin, 1999), ante un escenario complejo, para encaminar hacia el resultado esperado será necesario considerar la ética como factor multidimensional de la realidad, siendo que debe formarse en las mentes, a partir de la conciencia de que el humano es al mismo tiempo individuo, parte de una sociedad, parte de una especie. Por lo citado anteriormente se puede indicar que estos escenarios complejos al estar envueltos de certezas e incertidumbres debido a su impredicibilidad por una multiplicidad de factores subyacentes existentes los cuales emergen por infinitas causas que van cambiando constantemente, si no son analizados a profundidad de manera que eliminen el reduccionismo, constantemente se seguirá incurriendo en errores. Por tanto, para retomar los diversos acuerdos, de manera que permitan religar los conocimientos separados es necesario promover la capacidad de despliegue de la inteligencia humana, de manera que se pueda demandar y a su vez, proporcionar una nueva reforma del entendimiento.

\section{Chile}

La historia en relación al enclaustramiento de Bolivia ventila grandes conflictos tanto para Bolivia como para Chile y toda la región y sobre todo tienen un profundo significado psicológico, cultural y social. Desde que dio inicio a su mandato, el presidente Evo Morles asentó como prioridad, realizar gestiones para lograr la tan anhelada salida al océano pacífico y la refundación del estado. Según (Vera, 2016) la insistencia del gobierno de Evo Morales desde el año 2006, dio paso a una demanda internacional inédita que tiene como foco la idea que los actos unilaterales de Chile, en pos de poner sobre la mesa la discusión sobre la soberanía, obligando a Chile a una negociación pronta y de buena fe sobre la materia. Por este motivo, analizar Bolivia en el orden interestatal con su vecino Chile, significa ubicarse en el paradigma institucionalista de las relacione internacionales, o bien, bajo el paradigma neorrealista de las relaciones internacionales. Luego de tantos años y luego de que Chile despojara 
los puertos soberanos al océano pacífico, denota claramente un perjuicio económico ya que el flujo comercial no alcanza el nivel de desempeño esperado en comparación con otros países sudamericanos ya que Bolivia es uno de los países que menos está exportando.

Historiológica académica para la integración y solución del tema marítimo. Formas de resolver las controversias internacionales

Junto con la obligación o dicho de mejor forma, al compromiso de hallar una salida pacífica al océano, ante la multiplicidad de diferencias internacionales, está el principio de la libre elección de los métodos de resolución de controversias, que está íntimamente ligado al principio de soberanía de los Estados y de su igualdad jurídica (Cornejo, 2009). Es así que, para hacer frente a todas estas vicisitudes, será necesario considerar el enfoque transdisciplinario como refiere (Nicolescu, 1996) cuando se refiere a redescubrir y develar este concepto con una velocidad fulminante como consecuencia de un acuerdo necesario y con los desafíos de nuestro convulsionado mundo para descubrir la resurrección del sujeto y el principio de una nueva etapa de nuestra historia. Por lo citado, se puede decir que ningún Estado está sometido a otro dado que todo Estado es soberano. "Par in parem non habet imperium" Es entonces que los Estados están facultados a elegir, dentro de los mecanismos del Derecho Internacional, aquel que más les guste o convenga, tal como dispone la Resolución 2625 de la Asamblea General de la ONU al determinar que: "el arreglo de las controversias internacionales se basará en la igualdad soberana de los Estados y se hará conforme al principio de la libre elección de los medios" (ONU, 2010) Para ello cada estado puede elegir los mecanismos diplomáticos o no jurisdiccionales, como la negociación, la conciliación y mediación internacional. El desarrollo acelerado del enfoque transdisciplinario, debe ser considerado en el entendido de ser un nuevo movimiento de ideas y del peligro de multiplicidad de extravíos ya sean los mismos comerciales o de la búsqueda de nuevos medios de dominación del otro o como dice (Nicolescu, 1996), de verter la nada en el vacío a través de una adopción de un eslogan "bon ton" vaciado de todo contenido para re inventarse permanentemente. 


\section{CONCLUSIONES}

\section{Planteamiento de incertidumbres y certezas}

Por todo lo anteriormente expuesto en el presente artículo, en el tema marítimo entre

\section{Bolivia y chile se puede decir que:}

- Bolivia y Chile tienen pendiente una negociación, y deben abrir sus comunicaciones y dialogar, sin prisa pero sin pausas e interrupciones, de manera efectiva, sincera y con espíritu conciliador, hasta lograr un acuerdo mutuamente satisfactorio, lo cual solo sucederá cuando Bolivia establezca una política de Estado que integre los diferentes niveles de conducción y factores de poder a través de una defensa multidisciplinaria con un enfoque integrado de las dimensiones de poder por medio de la reflexión y la acción para lograr ir entre, desde, a través de y más allá de cualquier ideología y juntos desde la multiplicidad de posiciones, confluir en un proyecto futuro que permita construir, de construir y reconstruir a partir de hacer surgir lo nuevo y lo positivo.

- La Constitución de Bolivia señala que el país no renunciará a recuperar una salida marítima "con soberanía". por lo que se tiene que hacer un estudio complejo transdisciplinar de la realidad actual y dados los acontecimientos acaecidos en la Haya, realizar políticas y estrategias para emplear su poder Blanco en la persuasión de la población del norte de Chile que, dadas las circunstancias, vive del comercio y de las relaciones con Bolivia por lo cual tiene una afinidad socio cultural y de adaptación, incluso se recuerda que en alguna ocasión se izaron banderas Bolivianas en protesta a las políticas de Chile en la atención de esta región. Esta disyunción de pensamientos existente entre ambos países, desemboca en un paradigma profundo de simplificación el cual, ante cualquier complejidad conceptual, prescribe al ser de implicación/distinción/conjunción ya que permitirá la concepción de impedir concebir la realidad humana y la relación a la vez de implicación y separación entre el hombre y la naturaleza. Según (Morin, 1999), el paradigma es inconsciente, pero irriga el pensamiento consciente, lo controla $y$, en este sentido, es también sub consciente.

Según (Lacoste, (2016), desde una perspectiva crítica, se percibe que tanto Bolivia como Chile han abordado el conflicto con enfoques nacionalistas, que tienden a demonizar o minimizar al otro, sin capacidad de diálogo ni de construir alternativas 
de consenso. Se ha creado así, una suerte de enfrentamiento discursivo entre ambas naciones, sin mayores perspectivas de solución política.

\section{Referente a otras opciones de salida al Océano, después de desarrollado el análisis} complejo se puede decir lo siguiente:

- En el tema de la salida por puerto Buch con países como Paraguay, Brasil y Argentina, Bolivia se convertiría en un pivote entre el Atlántico y el Pacífico y, además, a partir de su condición de segunda potencia gasífera del continente, podría consolidarse como una reserva energética en la región, el descubrimiento del gas Boliviano el cual ha sido el origen de nuevas instancias de acercamiento entre varios países de la región. Hoy en día la exploración de nuevos mercados del litio hace que intereses internacionales estén con miras a este producto. Esta desmitificación desde el enfoque complejo y transdisciplinar, permitirá instaurar relaciones primordiales considerando los discursos y/o teorías, organizando la organización de los mismos generando la generación o la regeneración de ideas que confluyan en un beneficio sujeto-objeto, por un lado, un sujeto con ideologías y problemas de existencia y por el otro, objetos sometidos a observaciones, experimentaciones y manipulaciones, pudiendo así dilucidar y cegar, revelar y ocultar.

- Referente a las otras posibilidades ya mencionadas en lo que concierne al Puerto Ilo y Puerto Buch, se deben realizar las gestiones de gobierno para desarrollar políticas de Estado que garanticen la prosecución de acciones para logar el desarrollo de estas y que se conviertan en el polo de desarrollo para nuestro Estado. Por tanto, todas las determinaciones sociales, económicas, políticas y culturales deberán converger y sinergizarse para encarcelar al conocimiento en un multideterminismo de imperativos, normas, prohibiciones, rigideces y bloqueos. Reconociendo estas realidades recién podremos avanzar en un diálogo que permita eliminar los conformismos cognitivos e intelectuales. Desde el punto de vista de la complejidad, se puede analizar el contexto lógico de manera que se pueda concebir unidad y diversidad, orden y desorden para que, a partir del conflicto, se tienda a producir algo nuevo.

En consecuencia, será necesaria eliminar esa ideología homicida del ser humano para eliminar el reduccionismo de pensar solo en el propio beneficio, impulsando la reflexión y la conciencia de los múltiples actores que constantemente van variando para confluir 
como dice (Morin, 1999) en "establecer una relación de control mutuo entre la sociedad y los individuos o por medio de la democracia y concebir la humanidad como comunidad planetaria sin dejar de lado que no hay un estado superior de la razón que domine la emoción, sino, un bucle intellect- effect; y de cierta manera, la capacidad de emoción es indispensable para el establecimiento de comportamientos racionales.”.

Para confluir en acuerdos que consideren la unidualidad existente entre los elementos subyacentes para solucionar el tema marítimo entre Bolivia y Chile, será necesario que prime la racionalización que según (Morin, 1999) "se cree racional porque constituye un sistema lógico perfecto, basado en la deducción o la inducción; pero ella se funda sobre bases mutiladas o falsas y se niega a la discusión de argumentos y a la verificación empírica. La racionalización es cerrada y también abierta y toma las mismas fuentes de la racionalidad, pero constituye una de las fuentes de errores y de ilusiones más poderosa. La verdadera racionalidad, abierta por naturaleza, dialoga con una realidad que se le resiste". Por tanto, ese debe ser el gran desafío nos solo para ambos países sino, para la humanidad.

Finalmente, como dice (Morin \& Le Moigne, La inteligencia de la complejidad Epistemología y Pragmática, 2006): "Si aceptamos que hay problema de irreducibilidad, de indeducibilidad, de relaciones complejas entre las partes y el todo, y si además concebimos un sistema como una unidad compuesta de partes diferentes, estamos en la obligación de unir la noción de unidad con la pluralidad, o al menos de diversidad. Entonces nos percatamos de que hay que llegar a una complejidad lógica, pues tenemos que unir nociones que, por lo común y por lógica, se repelen, como la unidad y la diversidad e incluso el azar y la diversidad, el orden y el desorden tienen que combinarse para poder concebir la génesis de las organizaciones físicas, entonces estamos en la obligación de religar todas esas nociones separadas en el entendimiento que infortunadamente se nos inculcó desde la infancia”.

\section{REFERENCIAS BIBLIOGRÁFICAS}

AGPE. (1997). Defensa Nacional de Chile. Santiago de Chile: AGPE.

apertura, P. d. (2011). Cumbre de América Latina y del Caribe. III Cumbre de jefes de Estado. Caracas, Venezuela: http://constitucionweb.blogspot.com/2011/12/celac-discurso-de-evomorales.html. 
Cornejo, A. D. (2009). Bolivia y su reclamo a la salida al mar. Buenos Aires: IESE.

Cruz Barreiro, M. (2009). El papel de la educación en la identidad humana. Eikasia: Revista de filosofía, 5.

Defensa, M. d. (2004). Libro Blanco de Defensa. La Paz: IGM.

ERBOL. (23 de Marzo de 2021). Arce fija agenda de nueve puntos para la politica maritima. dia del mar, pág. 5 .

Lacoste, P. \&. ((2016). El enclaustramiento de Bolivia y el factor Argentina. Si Somos Americanos. Scielo, 119-145.

Longaric Rodriguez, K. (2014). Análisis Jurídico Sobre la Demanda Presentada por Bolivia Ante el Tribunal de Justicia de La Haya. La Paz-Bolivia: PDF. http://www.institutoprisma.org/joomla/images/DocumentosDeTrabajo/RR II/demanda\%20ante\%20La\%20Haya.pdf. La Paz Bolivia. Instituto Prisma.

Ministerio de Defensa de la Nación República Argentina. (2009). Manual para la identificación, formulación y evaluación de proyectos con inversión de la Defensa basados en capacidades. Buenos Aires: Ministerio de Defensa de la Nación República Argentina.

Moran, E. (2010). Introduccion al pensamiento complejo. Paris: Mound.

Morin, E. (1999). Los 7 saberes necesarios para la eduacaión del futuro. Paris- Francia: Santillana - UNESCO.

Morin, E., \& Le Moigne, J. (2006). La inteligencia de la complejidad Epistemología y Pragmática. México: Coloquio de Cerisy.

Nicolescu, B. (1996). La Transdisciplinariedad. México: Multiversidad Mundo Real de Edgar Morin.

OCCMUNDIAL. (19 de ENERO de 2021). OCCMUNDIAL. Obtenido de QUE ES UN ANALISIS FODA: https://www.occ.com.mx/blog/que-es-un-analisis-foda-ycomo-se-hace/

ONU. (2010). DERECHO INTERNACIONAL. NY: Strar Oest.

Presidencia del Gobierno de Espana. (2017). Estrategia de Seguridad Nacional 2017. Madrid: Presidencia del Gobierno de Espana.

RESDAL. (2011). Bases para una politica de defensa nacional. Red de seguridad y defensa para america latina, 2.

Tello, A. P. (2016). Conceptos de seguridad y defensa. GERI-UAM, 2. 
Valenzuela Espinoza, I. (2012). Complejidad, globalizacion y teoria social. Iquique: Revista Latinoamericana.

Vera, L. C. (2016). La disputa por el mar: El soft power boliviano ante organismos multilaterales. Revista de Relaciones Internacionales, Estrategia y Seguridad, 263-286. 